\title{
Real-time traffic sign detection and recognition using Raspberry Pi
}

\author{
Ida Syafiza Binti Md Isa', Choy Ja Yeong', Nur Latif Azyze bin Mohd Shaari Azyze² \\ ${ }^{1}$ Department of Electronics and Computer Engineering Technology (JTKEK), Faculty of Electrical and Electronic Engineering \\ Technology, Universiti Teknikal Malaysia Melaka (UTeM), Melaka, Malaysia \\ ${ }^{2}$ Department of Mechatronic Engineering, Faculty of Electrical Engineering, Universiti Teknikal Malaysia Melaka (UTeM), \\ Melaka, Malaysia
}

\section{Article Info \\ Article history: \\ Received Jan 23, 2021 \\ Revised Jun 21, 2021 \\ Accepted Aug 2, 2021}

\section{Keywords:}

Accuracy

Delay

Recognition

Reliability

Traffic sign

\begin{abstract}
Nowadays, the number of road accident in Malaysia is increasing expeditiously. One of the ways to reduce the number of road accident is through the development of the advanced driving assistance system (ADAS) by professional engineers. Several ADAS system has been proposed by taking into consideration the delay tolerance and the accuracy of the system itself. In this work, a traffic sign recognition system has been developed to increase the safety of the road users by installing the system inside the car for driver's awareness. TensorFlow algorithm has been considered in this work for object recognition through machine learning due to its high accuracy. The algorithm is embedded in the Raspberry Pi 3 for processing and analysis to detect the traffic sign from the real-time video recording from Raspberry Pi camera NoIR. This work aims to study the accuracy, delay and reliability of the developed system using a Raspberry Pi 3 processor considering several scenarios related to the state of the environment and the condition of the traffic signs. A real-time testbed implementation has been conducted considering twenty different traffic signs and the results show that the system has more than $90 \%$ accuracy and is reliable with an acceptable delay.
\end{abstract}

This is an open access article under the $\underline{C C B Y-S A}$ license.

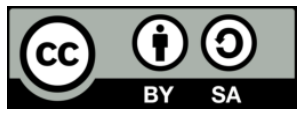

\section{Corresponding Author:}

Ida Syafiza Binti Md Isa

Department of Electronics and Computer Engineering Technology (JTKEK), Faculty of Electrical and Electronic Engineering Technology, Universiti Teknikal Malaysia Melaka (UTeM)

Hang Tuah Jaya, 76100 Durian Tunggal, Melaka, Malaysia

Email: idasyafiza@utem.edu.my

\section{INTRODUCTION}

Nowadays, the number of road accident in Malaysia is increasing expeditiously. As reported in [1], Malaysian has the third highest fatality rate of road accidents in ASEAN, with 7,512 Malaysian citizens' death in 2016. It also reported that the fourth most common cause of death for Malaysian people is road accidents. Traffic sign recognition system is a pivotal platform that can be implemented in the autonomous driving system. Sometimes, the driver may overlook the traffic sign due to tiredness, and this will indirectly cause road accidents [2]. Besides, the blockage of the traffic signs by big vehicle and tree also cause the driver to overlook the traffic sign. Hence, a traffic signs recognition system that is able to detect the traffic signs is necessary to alert the driver. The development of the traffic sign recognition system has been considered in the past few years to reduce the number of accidents. The first traffic sign detection and recognition (TSDR) system was developed in Japan since 1984 [3]. The TSDR system used computer vision with eight standard datasets recognition techniques, included German TSDR benchmark (GTSDRB), KUL 
Belgium traffic sign dataset (KULD), Swedish traffic signs dataset (STSD), The Netherlands RUG traffic signs database (RUGD), France Stereopolis database, United States LISA dataset (LISAD), United Kingdom online dataset (UKOD) and Russian traffic sign dataset (RTSD) for fast detection of the traffic signs. Figure 1 shows a sample of traffic signs.

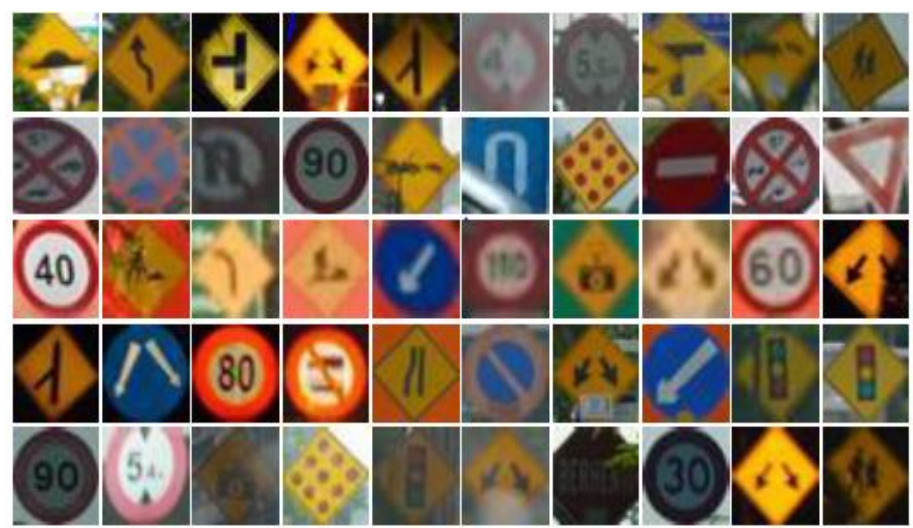

Figure 1. Dataset sample of traffic signs [3]

Advanced driving assistance system (ADAS) is a system developed by professional engineers with the aims to reduce the number of road accidents. This include an intelligent system to detect vehicles for traffic management purposes [4]-[6] and the traffic sign along the road [7]. However, the type of machine learning algorithm used in the traffic sign recognition system to efficiently detect the traffic sign is very important. The weather condition and the reflection of light on the traffic sign can reduce the quality of the captured traffic sign image [7]. Therefore, an efficient algorithm is required to perform the processing and analysis of the captured image. Several machine learning algorithm has been proposed for traffic sign recognition include the TensorFlow transfer learning algorithm [8]-[10], AdaBoost algorithm [11], convolutional neural networks (CCN) algorithm [12], [13], fuzzy integral algorithm [14], neural network [15], artificial neural network (ANN) [16], deep learning [17], [18], color transformation [19], and texture feature extraction [20]. The types of algorithms used inside TensorFlow, such as transfer learning, increase the efficiency of the traffic sign recognition when compared to traditional machine learning. Note that transfer learning is developed using information or knowledge from the surrounding environment [8]. The training and test samples are randomly selected, which can be a large amount of data or a small amount of labelled data. Transfer learning allows training only at the last layer of the whole Inception-v3 network, and this can help to reduce the training time and maintain the accuracy of recognition [21]. AdaBoost algorithm has been proposed to reduce the noise of the segmented traffic sign images. To increase the accuracy, the AdaBoost algorithm requires a big dataset of sample images to detect the traffic sign. According to [22], a $\mathrm{CNN}$ ensemble is used in the traffic sign smart detection system, which focuses on colour-based detection, shape-based detection and sign validation. According to [23], CNN can be used to annotate the sample images as $\mathrm{CNN}$ is a class of models that is easier to be trained with the sample images. Besides, a data segmentation method is also used in $\mathrm{CCN}$ to reduce the image data overfitting by performing the process of label-preserving transformation. However, the colour of the traffic sign and the lack of light during night may effected the performance of the CCN.

Meanwhile, several researchers considered Raspberry Pi, which has limited computation capability to develop the IoT-based application system [24]-[27]. As in [24], the authors used the Raspberry Pi to observe wildlife using deep learning, giving up to 97\% accuracy. Akshay and Dinesh in [25] study the shading effect on the road sign recognition system that runs on the Raspberry Pi. However, the performance of the system is not clearly reported. Kharkar [26] developed a road sign detection and recognition robot system using an edge detection algorithm running on the Raspberry Pi to detect the traffic sign. However, the performance of the developed system is not evaluated in a real environment which might introduce a significant impact (i.e. reflection of light) on the captured traffic sign image. Meanwhile, the authors in [27] developed a road sign recognition system using Raspberry Pi but only focused on the speed sign while considering the stability of colour detection due to daylight condition. The results show that the system has $80 \%$ accuracy with a processing delay of up to 2 seconds. 
In this work, a real-time traffic sign detection and recognition system using the TensorFlow machine-learning algorithm to process and analyze the sample model of traffic signs has been developed to assist the driver. The system used Raspberry Pi 3 that is embedded with the TensorFlow algorithm, to detect the type of traffic sign based on the real-time video recording obtained by Raspberry Pi camera NoIR and send alerts to the driver. The accuracy, the reliability and the delay of the developed system are evaluated and analyzed through a real-time testbed implementation in a real environment considering five different class of traffic signs, the condition of the traffic sign itself (i.e. blurred, and broken) and also the state of the environment.

\section{RESEARCH METHOD}

Raspberry $\mathrm{Pi}$ is a mini-computer that is capable of running applications as the same standard desktop computer [28]. In this work, a Raspberry Pi 3 module is used to run the TensorFlow machine learning algorithm and is connected to a Raspberry Pi camera NoIR to record the real-time video, a Raspberry Pi Display module to display the related information of the detected traffic sign and a speaker to give an alert sound to alert the driver. Figure 2 shows the complete hardware of the developed system. Note that the developed system will be deployed in a car on the dashboard and is powered using power car adapter.

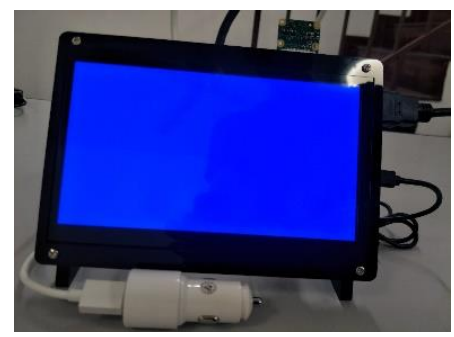

(a)

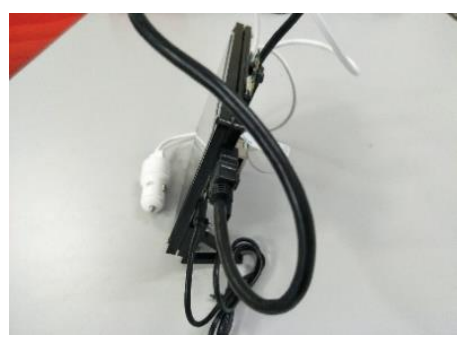

(b)

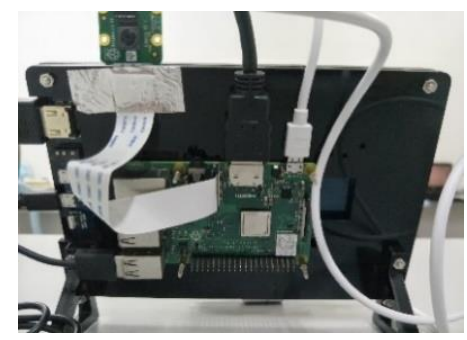

(c)

Figure 2. The developed real-time traffic sign detection and recognition system; (a) front view, (b) side view, and (c) rear view

Meanwhile, the working principles of the developed real-time traffic sign recognition system are as shown in Figure 3. First, the Raspberry Pi 3 and Raspberry Pi camera NoIR are in stand-by mode once the system is powered on. Then, the Raspberry Pi camera NoIR will start records the real-time video and share the video with the Raspberry Pi 3 synchronously. The Raspberry Pi 3 will process the video to detect the type of traffic sign. If a traffic sign is detected, the results will be sent to the Raspberry Pi Display Module to display the type of the detected traffic sign and activate the speaker. Otherwise, the camera will keep on recording and sending the data to the Raspberry Pi 3 module for processing until the traffic sign detected.

\subsection{Labelimg}

Labelimg is also known as label image, which is an open-source image labelling tool that is used to label the size of the traffic sign image dataset. Each traffic sign image needs to be labelled before starting the training process of the traffic sign images with TensorFlow machine learning software. The purpose of labelling the traffic sign images is to determine the location of the traffic signs. Besides, Labelimg carries out the process of segmentation of traffic sign images and then continues with the process of annotation and interpretation for the traffic sign images. Annotation of traffic sign images is important as the bounding outside boxes will be shown on the traffic sign images, thus the images will be easily recognized, as shown in Figure 4. The coding used for Labelimg is Python programming language. After Labelimg annotates the sample set of traffic sign images, it saves the images as ' $\mathrm{XML}$ ' file format, and the images are ready to be trained and tested by TensorFlow.

\subsection{TensorFlow machine learning}

As stated above, in this work, the TensorFlow machine learning algorithm is used to train a dataset consisting of five different classes of traffic signs, including the Speed Bump, Stop, Give Way, No U-Turn and Chevron Alignment. These five classes of traffic signs are considered as they are commonly found at the roadside [29]. For each class, there are 100 sample images with different angles, and size hence give a total of 500 sample images. The training process is important to prepare the pre-trained model before the implementation of the real-time recognition system. The pre-trained model that contained the size, colour, shape and boundary of the sample traffic sign images is used for the recognition. The flowchart of the 
TensorFlow algorithm is as shown in Figure 5. In this traffic sign recognition system, TensorFlow Lite version 2.5.0 machine learning software is installed on the Raspberry Pi 3 to run the pre-trained model, which consists of the dataset that was trained before. For the traffic sign recognition, the Raspberry Pi 3 will run the TensorFlow algorithm to detect the traffic signs based on the real-time video recording from the Raspberry Pi camera NoIR with the help of the Labelimg results performed for traffic sign classification as discussed in section 2.1. Once the traffic sign is detected, the Raspberry Pi Display Module will show the type of that traffic sign with the percentage of its accuracy. Figure 6 shows the coding to run TensorFlow software on object recognition.

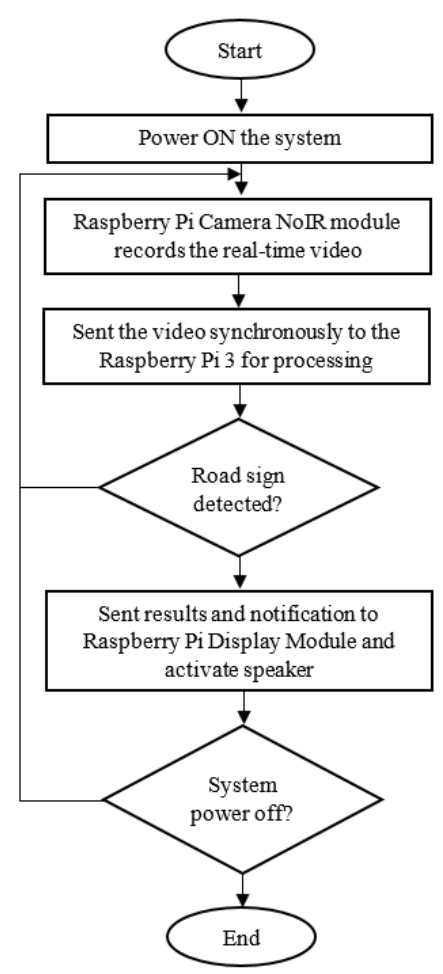

Figure 3. The flow system of the real-time traffic sign detection and recognition system algorithm

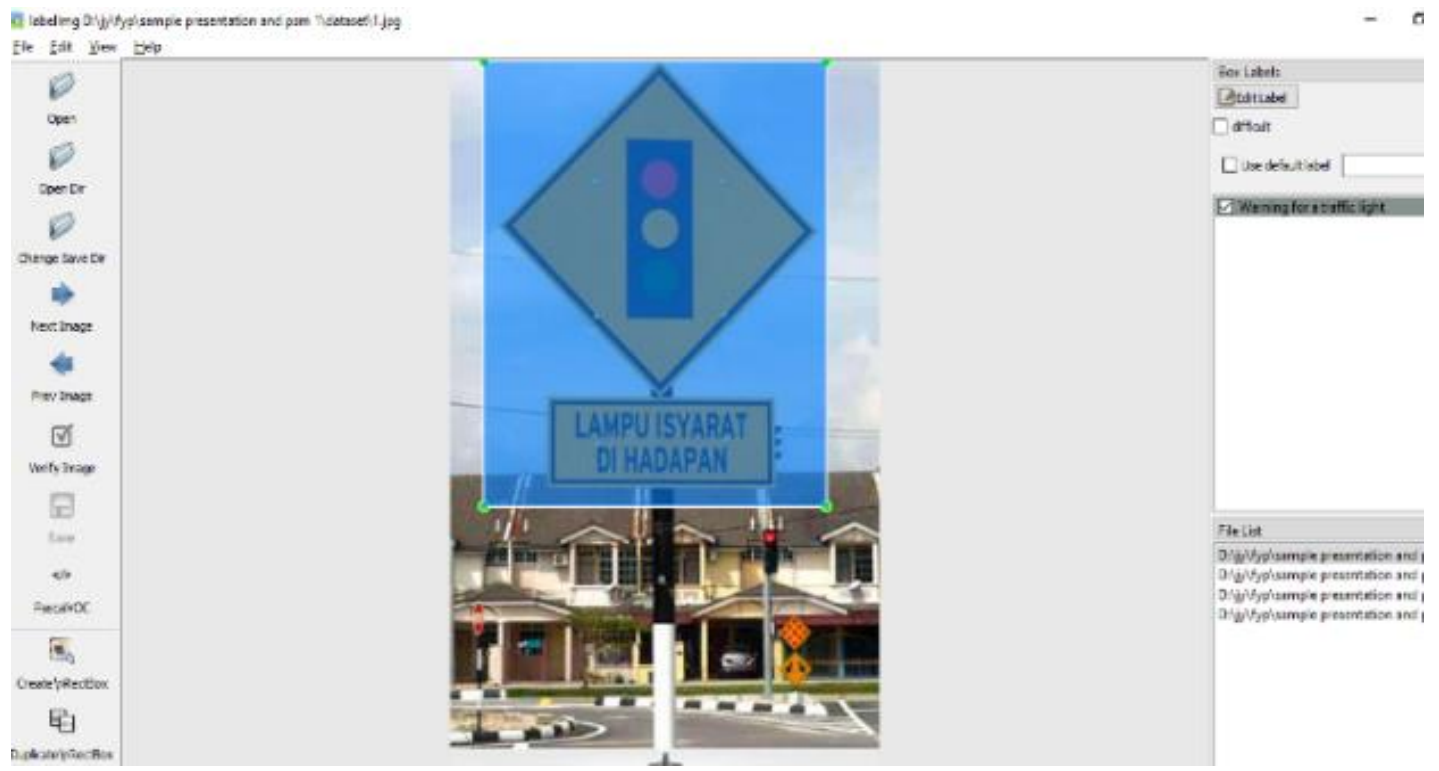

Figure 4. Labelling the traffic sign sample image 


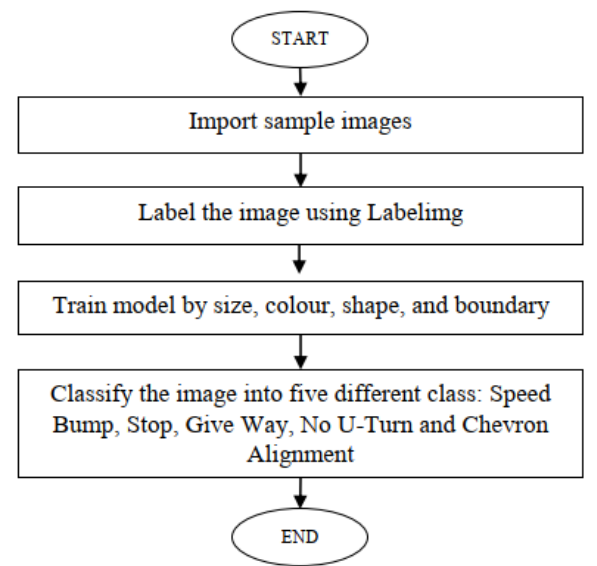

Figure 5. Flowchart of TensorFlow machine learning image classification

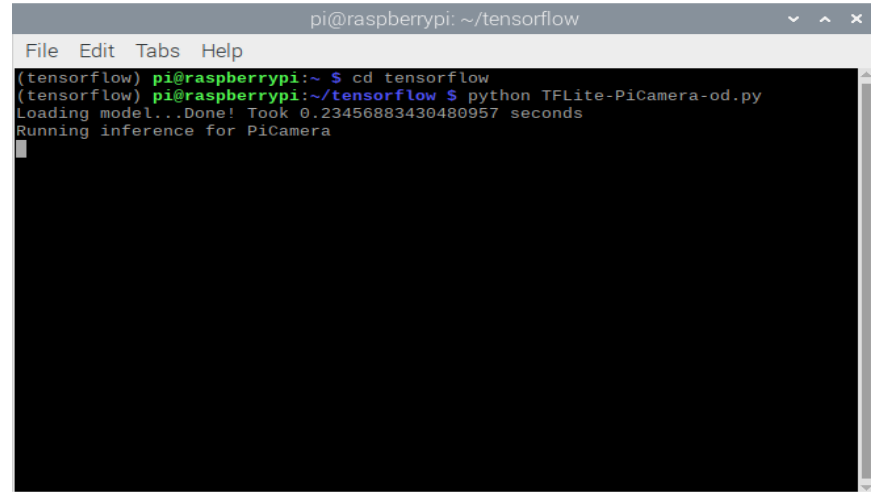

Figure 6. Coding for starting detection using TensorFlow machine learning

\section{RESULTS AND DISCUSSION}

In this work, the performance of the developed real-time traffic sign detection and recognition system in terms of accuracy, reliability and delay has been evaluated considering five different classes of traffic signs, the state of the environment and the condition of the traffic sign. The developed system is installed in a car, and the speed of the car considered in this experiment is 50 kilometres per hour $(50 \mathrm{~km} / \mathrm{h})$. Note that the locations considered to perform the testbed implementation of the developed system are at Taman Muzaffar Height, Ayer Keroh and Kawasan Perindustrian Ayer Keroh, in which the five classes of traffic signs considered in this work are available. Figure 7 shows the samples images that are displayed on the Raspberry Pi display module when the traffic sign is detected during the experiment. Note that the percentage of the accuracy of the detected traffic sign is also displayed on the Raspberry Pi display module.

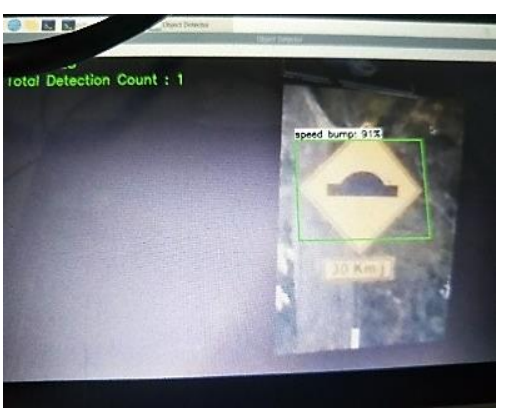

(a)

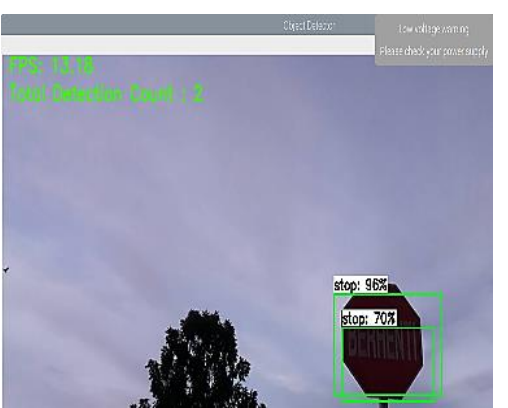

(b)

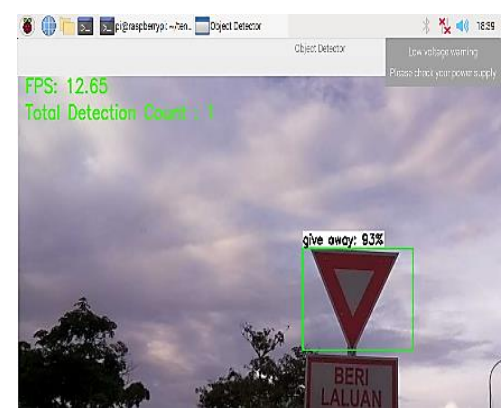

(c)

Figure 7. Screenshot of (a) speed bump, (b) stop, and (c) give way, traffic sign is recognized from the front view 


\subsection{System accuracy}

To evaluate the accuracy of the developed system, 20 traffic sign images from the five different classes of traffic signs are captured during the testbed implementation. Note that the testbed implementation is performed during normal weather condition at a speed car of $50 \mathrm{~km} / \mathrm{h}$. Figure 8 shows the average percentage of the accuracy of the five classes of the traffic sign. The results show that the average percentage of the accuracy for all classes of traffic sign image is above $90 \%$. This indicates that the developed system works efficiently to detect the traffic sign images and identify the type of traffic sign based on real-time video recording.

\subsection{Delay}

The performance of the developed system in term of delay (i.e. processing time) is also evaluated for the 20 traffic sign images from the five different classes of traffic sign captured during the testbed implementation. The delay refers to the time taken to load and read the pre-trained model when the real-time video is running. Note that a normal speed of the car, which is $50 \mathrm{~km} / \mathrm{h}$ in normal weather, is considered during the testbed implementation. Figure 9 shows the average time delay in the developed system to detect and identify the type of traffic sign images from the five traffic sign classes. The results show that the maximum average delay is 3.44 seconds while the minimum is 3.23 seconds. This shows that the developed recognition system is suitable for the city speed limit of the car. However, it is not recommended for a fastmoving car (i.e. above $50 \mathrm{~km} / \mathrm{h}$ ) to avoid the latency in the system, hence endanger the driver.

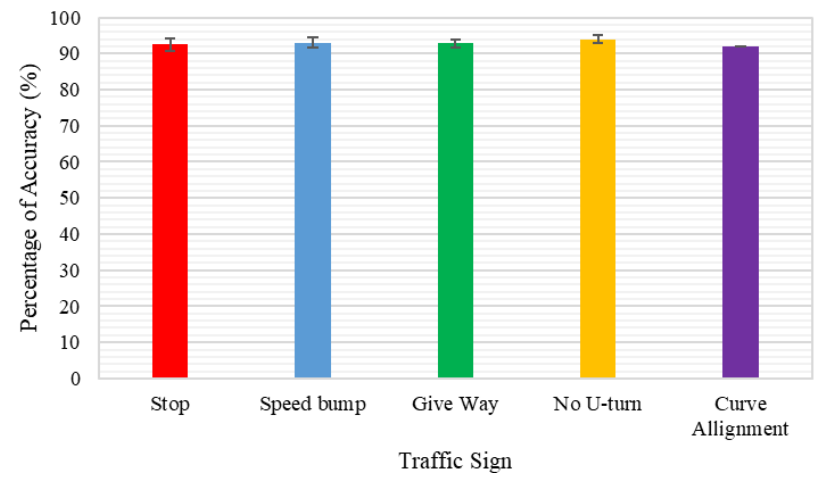

Figure 8. Percentage of accuracy for different classes of traffic sign

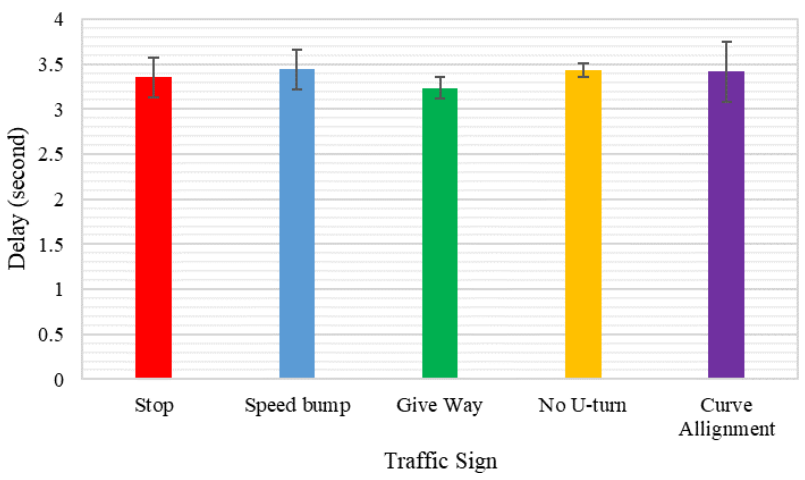

Figure 9. Delay for different classes of traffic sign

\subsection{Reliability}

The reliability of the developed real-time traffic sign recognition system is evaluated considering the broken traffic sign such as the incomplete part of the traffic sign or the faded traffic sign and the lighting at the testbed implementation (i.e. at night-time). Figure 10(a)-(c) show the broken traffic sign image with its detected percentage of accuracy, the detected traffic sign images during night-time with its percentage of accuracy, and the considered faded traffic sign with its percentage of accuracy, respectively. For all scenarios, the results show that the developed system able to perform well with an accuracy of the traffic sign signal above $90 \%$. 


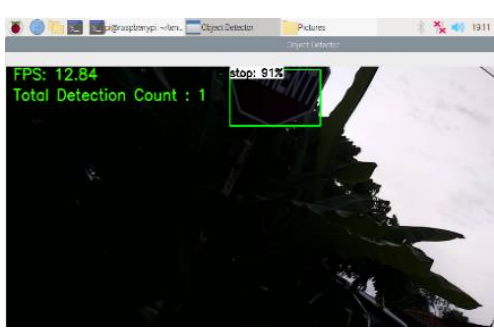

(a)

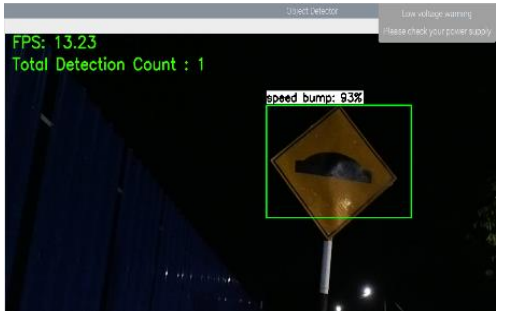

(b)

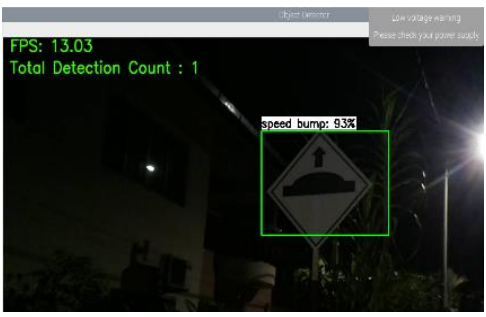

(c)

Figure 10. The traffic sign detection considering; (a) incomplete traffic sign, (b) night-time condition, and (c) faded traffic sign with the percentage of accuracy

\section{CONCLUSION}

In this work, a real-time alert system for traffic sign recognition system is developed using the Raspberry Pi 3 processor. The TensorFlow machine learning algorithm is used to train the traffic sign and detect the traffic sign through the real-time recording video using Raspberry Pi camera NoIR to alert the drivers. Twenty traffic sign images from five different classes of traffic sign have been considered for the real-time testbed implementation. The performance of the developed system has been evaluated in terms of accuracy, delay and reliability. The results show that the average accuracy of detecting and identifying traffic sign images from the five traffic sign classes is above $90 \%$.

Meanwhile, the results also show that the maximum average delay in determining the type of traffic sign in the system is 3.44 seconds when the car moves at $50 \mathrm{~km} / \mathrm{h}$. This shows that the system is suitable to be used for a city speed limit of the car. In term of reliability, the developed system is tested considering the condition of the traffic sign such as broken and blurred traffic sign and the state of the environment (i.e. during night-time). The results indicate that the developed system is reliable to detect the type of traffic sign with $90 \%$ accuracy for all considered conditions and send out an alert to the drivers.

\section{ACKNOWLEDGEMENTS}

Authors would like to thank Centre of Research and Innovation Management (CRIM), Universiti Teknikal Malaysia Melaka (UTeM), and Ministry of Education, Malaysia for supporting this research.

\section{REFERENCES}

[1] M. Lum, "We have the third highest death rate from road accidents," The Star Online, pp. 1-10, 2019.

[2] C. F. Cheah, "Microsleep a factor in road accidents," nst.com.my. https://www.nst.com.my/opinion/letters/2020/07/607009/microsleepfactor-road-accidents (accessed Jan. 23, 2021).

[3] A. Madam and R. Yusof, "Malaysian traffic sign dataset for traffic sign detection and recognition systems," Journal of Telecommunication, Electronic and Computer Engineering, vol. 8, no. 11, pp. 137-143, 2016.

[4] M. Anandhalli, V. P. Baligar, P. Baligar, P. Deepsir, and M. Iti, "Vehicle detection and tracking for traffic management," IAES International Journal of Artificial Intelligence (IJ-AI), vol. 10, no. 1, pp. 66-73, 2021, doi: 10.11591/ijai.v10.i1.pp66-73.

[5] M. Anandhalli and V. P. Baligar, "An approach to detect vehicles in multiple climatic conditions using the corner point approach," Journal of Intelligent Systems, vol. 27, no. 3, pp. 363-376, 2018, doi: 10.1515/jisys-2016-0073.

[6] M. Anandhalli and V. P. Baligar, "A novel approach in real-time vehicle detection and tracking using Raspberry Pi," Alexandria Engineering Journal, vol. 57, no. 3, pp. 1597-1607, 2018, doi: 10.1016/j.aej.2017.06.008.

[7] S. B. Wali et al., "Vision-based traffic sign detection and recognition systems: Current trends and challenges," Sensors, vol. 19, no. 9, 2019, doi: 10.3390/s19092093.

[8] X.-L. Xia, C. Xu, and B. Nan, "Facial expression recognition based on tensorflow platform," ITM Web of Conferences, vol. 12, pp. 1-4, 2017, Art. no. 01005, doi: 10.1051/itmconf/20171201005.

[9] A. Benhamida, A. R. Varkonyi-Koczy, and M. Kozlovszky, "Traffic signs recognition in a mobile-based application using tensorflow and transfer learning technics," SOSE 2020 - IEEE 15th International Conference of System of Systems Engineering Proceedings, 2020, pp. 537-541, doi: 10.1109/SoSE50414.2020.9130519.

[10] I. Kilic and G. Aydin, "Traffic sign detection and recognition using tensorflow' s object detection API with a new benchmark dataset," 2020 International Conference on Electrical Engineering ICEE, 2020, pp. 000537-000542, doi: 10.1109/ICEE49691.2020.9249914.

[11] Y. Saadna and A. Behloul, "An overview of traffic sign detection and classification methods," International Journal of Multimedia Information Retrieval, vol. 6, no. 3, pp. 193-210, 2017, doi: 10.1007/s13735-017-0129-8.

[12] X. Changzhen, W. Cong, M. Weixin, and S. Yanmei, "A traffic sign detection algorithm based on deep convolutional neural network," 2016 IEEE International Conference on Signal and Image Processing ICSIP, 2017, pp. 676-679, doi: 10.1109/SIPROCESS.2016.7888348.

[13] M. T. Islam, "Traffic sign detection and recognition based on convolutional neural networks," 2019 6th IEEE International Conference on Advances in Computing, Communication and Control ICAC3, 2019, pp. 2851-2854, doi: 10.1109/ICAC347590.2019.9036784. 
[14] C. Taştimur, M. Karaköse, Y. Çelik, and E. Akin, "Image processing based traffic sign detection and recognition with fuzzy integral," International Conference on Systems, Signals, and Image Processing, vol. 2016, 2016, pp. 6-9, doi: 10.1109/IWSSIP.2016.7502715

[15] A. Avramovic, D. Sluga, D. Tabernik, D. Skocaj, V. Stojnic, and N. Ilc, "Neural-network-based traffic sign detection and recognition in high-definition images using region focusing and parallelization," IEEE Access, vol. 8, pp. 189855-189868, 2020, doi: 10.1109/access.2020.3031191.

[16] M. Z. Abedin, P. Dhar, and K. Deb, "Traffic sign recognition using hybrid features descriptor and artificial neural network classifier," 19th International Conference on Computer and Information Technology ICCIT, 2017, pp. 457-462, doi: 10.1109/ICCITECHN.2016.7860241.

[17] C. Wang, "Research and application of traffic sign detection and recognition based on deep learning," Proceedings - 2018 International Conference on Robots and Intelligent System ICRIS, 2018, pp. 150-152, doi: 10.1109/ICRIS.2018.00047.

[18] D. Tabernik and D. Skočaj, "Deep learning for large-scale traffic-sign detection and recognition," IEEE Transaction Intelligent Transportation System, vol. 21, no. 4, pp. 1427-1440, 2019, doi: 10.1109/TITS.2019.2913588.

[19] M. B. Mahatme and S. Kuwelkar, "Detection and recognition of traffic signs based on RGB to red conversion," Proceedings of the International Conference on Computing Methodologies and Communication ICCMC, vol. 2018, 2018, pp. 447-451, doi: 10.1109/ICCMC.2017.8282728.

[20] C. Rahmad, I. F. Rahmah, R. A. Asmara, and S. Adhisuwignjo, "Indonesian traffic sign detection and recognition using color and texture feature extraction and svm classifier," 2018 International Conference on Information and Communications Technology ICOIACT, 2018, vol. 2018, pp. 50-55, doi: 10.1109/ICOIACT.2018.8350804.

[21] C. Lin, L. Li, W. Luo, K. C. P. Wang, and J. Guo, "Transfer learning based traffic sign recognition using inception-v3 model," Periodica Polytechnica Transportation Engineering, vol. 47, no. 3, pp. 242-250, 2019, doi: 10.3311/PPtr.11480.

[22] A. Vennelakanti, S. Shreya, R. Rajendran, D. Sarkar, D. Muddegowda, and P. Hanagal, "Traffic sign detection and recognition using a CNN ensemble," 2019 IEEE International Conference on Consumer Electronics ICCE, 2019, pp. 37-40, doi: 10.1109/ICCE.2019.8662019.

[23] A. Krizhevsky, I. Sutskever, and G. E. Hinton, "ImageNet classification with deep convolutional neural network," Advances in neural information processing systems, vol. 25, pp. 1097-1105, 2012, doi: 10.1145/3065386.

[24] B. H. Curtin and S. J. Matthews, "Deep learning for inexpensive image classification of wildlife on the Raspberry Pi," 2019 IEEE 10th Annual Ubiquitous Computing, Electronics and Mobile Communication Conference, UEMCON, 2019, pp. 0082-0087, doi: 10.1109/UEMCON47517.2019.8993061

[25] N. G. Akshay and K. Dinesh, "Road sign recognition system using raspberry pi," International Journal of Pure and Applied Mathematics, vol. 119, no. 15, pp. 1845-1850, 2018.

[26] V. P. Kharkar, "A road sign detection and recognition robot using raspberry-pi," International Research Journal of Engineering and Technology, vol. 5, no. 9, pp. 1001-1005, 2018.

[27] E. Bilgin and S. Robila, "Road sign recognition system on raspberry pi," 2016 IEEE Long Island Systems, Applications and Technology Conference LISAT, 2016, pp. 1-5, doi: 10.1109/LISAT.2016.

[28] S. J. Johnston and S. J. Cox, "The raspberry pi: a technology disrupter, and the enabler of dreams," Electronics, vol. 6, no. 3, 2017, doi: 10.3390/electronics6030051.

[29] Zumar. "Common highway signs and why they are important." zumar.com. 2021. https://www.zumar.com/blog/commonhighway-signs-and-why-they-are-important (accessed May 22, 2021). 